\title{
Effects of Coconut Husk and Corn Cob as Fillers in Flexible Polyurethane Foam
}

\author{
Pauline Uchechukwu Chris-Okafor ${ }^{1}$, Arinze Rose Mary Uchechukwu ${ }^{1}$, Joy Nwando Nwokoye ${ }^{2}$, \\ Ernest Umar Ukpai ${ }^{1}$ \\ ${ }^{1}$ Department of Pure \& Industrial Chemistry, Nnamdi Azikiwe University, Awka, Nigeria \\ ${ }^{2}$ Department of Chemistry, Federal College of Education Technical, Umunze, Nigeria
}

Email address:

pu.ofora@unizik.edu.ng (P. U. Chris-Okafor)

\section{To cite this article:}

Pauline Uchechukwu Chris-Okafor, Arinze Rose Mary Uchechukwu, Joy Nwando Nwokoye, Ernest Umar Ukpai. Effects of Coconut Husk and Corn Cob as Fillers in Flexible Polyurethane Foam. American Journal of Polymer Science and Technology.

Vol. 3, No. 4, 2017, pp. 64-69. doi: 10.11648/j.ajpst.20170304.12

Received: May 1, 2017; Accepted: June 28, 2017; Published: July 25, 2017

\begin{abstract}
The effects of local materials as fillers incorporated into the flexible polyether foam recipes were investigated. The fillers; coconut husk and corn cob of mesh sizes of $150 \mu \mathrm{m}$ respectively were mixed in the ratio of 50:50. Varying percentages of the mixed fillers, $5 \%, 10 \%, 15 \%, 20 \%$ and $25 \%$ were mixed with polyether foam recipes in the appropriate formulations and physico-mechanical tests were carried out on the samples. Density and compression set showed an increasing trend with increase in the filler. Elongation at break and tensile strength showed a decrease in the value as the filler load was increasing. Hardness showed a slight random variation in the value as the filler load increased. Thermal conductivity also showed a decrease in its value as the filler load increased that means it can serve as a fire retardant. All these properties were compared to a controlled sample (sample without filler). These fillers can be used in the production of polyurethane foams since they are organic materials, thus they can enhance the biodegradability of polyurethane products and be use also as flame retardants.
\end{abstract}

Keywords: Coconut Husk, Corn Cob, Polyether Foam, Mechanical Properties

\section{Introduction}

Polyurethane foam products are common everywhere. Twenty four hours of the day, we live with it, it is in the car we drive, it is underneath the carpet we walk on; we sit on it in our living rooms, even when we sleep. It is difficult to imagine someone going through a day without coming in contact with some foam products. People sleep on foam mattresses, lay heads on foam pillows, use foam sheeting in furniture manufacture, incorporate shaped foam into dress making (shoulder pads) and use specialized foams for partitioning and insulation [1]. The use of flexible urethane foams for cushions, furniture and automobiles has displaced rubber foam in these applications because of improved strength, lower density and easier fabrication [2].

In general, industries that produce flexible polyurethane foams use fillers to modify the material's properties in some way, such as: dimensional stability, retraction from the mold and density [3-4]. When adding filler to a polymer to form a conjugated biphasic material, the tension applied to the polymeric matrix will be transferred in part to the disperse phase, the filler, since it presents properties superior to the pure polymer [5]. Efficient reinforcement is achieved by interactions of the filler polymer matrix via mechanisms of adhesion, which could be: adsorption, electrostatic attraction, chemical bonding and mechanical adhesion [6-7]. In flexible polyurethane foams, the fillers promote an increase in density and resistance to compression. However, they reduce the resiliency and contribute to the increase in permanent deformation. In addition, properties such as tear strength, for example, are significantly affected by the introduction of filler. Accordingly, it is necessary to know the end-use of the material in order to use the correct concentration in the polymer matrix, obtaining a product of reliable quality.

The interest in natural fibre reinforced composites is growing rapidly due to the high performance in mechanical properties. There are different types of natural fibres like coir fibre, sisal fibre, banana fibre, pineapple fibre etc. Fibres 
obtained from various parts of the plants are known as vegetable fibres [2]. Natural fibre composites are very cost effective materials, especially in building and construction, packaging, automobile and railway coach interiors and devices [3].

Since, the issue of environmental sustainability and climate change has become a major consideration in the development of new materials in the world [8]. Thus, these materials used as fillers are agricultural wastes, readily available and relatively inexpensive that they can lower resin costs, improve stiffness and act as environmental friendly way to reduce the use of petroleum-based plastics.

Therefore, there is a need to find a means of reducing the cost and improve foam properties. This project is aimed at on how to reduce foam cost and produce environmental friendly foam by using locally available fillers precisely coconut husk and corn cob.

\section{Materials and Methods}

\subsection{Preparation of Raw Materials}

The fillers were locally and randomly collected from
Iyiagu Estate, Awka, Anambra State, Nigeria. The collected samples were washed properly with water and sun dried completely for 7 days. The dried coconut husk and corn cob were ground using an electric mill and the powders were sieved respectively using an electronic sieve of mesh size $150 \mu \mathrm{m}$ for each. The powders were then mixed together at the ratio of 50:50. Polyether polyol and toluene diisocyanate, (TDI) were weighed out in their different quantities using triple beam balance and then poured into different beakers. Dimethylethylamine, (DMEA), stannous octate, silicone oil and water were measured using syringes for accurate stoichiometric reaction. The accurate required quantities of the filler samples were also weighed using the triple beam balance. The measurements of these materials were all based on parts per hundred, (pph) of polyol which can be arbitrary chosen from the various formulation range as in Table 1.

\subsection{Foam Formulation}

This is a list that shows the various chemicals/materials and their relative proportions required in the preparation of the foam samples. This is formed based on the density and other properties of the desired given foam.

Table 1. Foam formulation.

\begin{tabular}{lllllll}
\hline MATERIAL & X & A & B & C & D \\
\hline POLYOL (G) & 600 & 600 & 600 & 600 & 600 \\
TDI (G) & 330 & 330 & 330 & 330 & 330 \\
AMINE (ML) & 2 & 2 & 1 & 1 & 2 & 2 \\
STANNOUS OCTATE (ML) & 1 & 5 & 5 & 5 & 1 & 28 \\
SILICONEOIL (ML) & 5 & 28 & 28 & 1 & 5 \\
WATER (ML) & 28 & 5 & 10 & 28 & 28 \\
FILLER (G) & 0 & & & 25 \\
\hline
\end{tabular}

\subsection{Preparation of Flexible Polyether Foam}

The measured quantity of polyol was poured into the mixing bowl followed by the addition of the weighed quantity of filler and this was thoroughly stirred for about 1-2 minutes. After this, other measured quantities of reagents in the syringes such as silicone oil, DMEA, stannous and water were simultaneously poured into the mixing bowl containing the polyol with continuous and thorough stirring of the mixture which lasted for about 3-4 minutes before complete polymerization (nucleation) was achieved. At this point, the foam mixture was absolutely shiny and creamy showing complete nucleation of the reagent. Lastly, the measured quantity of TDI was rapidly poured into the foam mixture with stirring interrupted. After the addition of TDI, the stirring lasted for about 40-65 seconds when a sign of rising of the foam mixture was noticed and the rising slurry foam mixture was smartly poured into the prepared cuboid mould (carton box $8 \times 8 \times 8$ ) coated with silicone paper. The foam mixture was allowed to cure for three days [9].

\subsection{Characteristics of the Foam Samples}

The foam samples produced in this work were only characterized for the physico-mechanical and flammability properties.

\subsubsection{Mechanical Tests}

The following mechanical properties of the foam samples were determined using standard methods: density, compression set, hardness [10] while tensile strength and elongation at break were measured according to the standard specifications [11].

\subsubsection{Thermal Conductivity}

Each foam sample produced was cut into three pieces and the initial and final temperature ( $\mathrm{Ti}$ and $\mathrm{Tf}$ ) of the pieces were noted using a thermometer. The thermal conductivity of the foam sample were determined by adding up the differences between $\mathrm{Tf}$ and $\mathrm{Ti}$ of the pieces of sample and dividing all by 3 and multiplying by 100. It is expressed mathematically as

$$
\text { Thermal conductivity }=\frac{(\mathrm{Tf}-\mathrm{Ti}) 1+(\mathrm{Tf}-\mathrm{Ti}) 2+(\mathrm{Tf}-\mathrm{Ti}) 3 \times 100}{3}
$$




\section{Results and Discussion}

Table 2. Values for Density Test.

\begin{tabular}{llll}
\hline & VOLUME $\left(\mathbf{M}^{\mathbf{3}}\right)$ & WEIGHT $(\mathrm{KG})$ & DENSITY $\left(\mathbf{K G} / \mathbf{M}^{\mathbf{3}}\right)$ \\
\hline 0 & 0.002150 & 0.04786 & 22.26 \\
5 & 0.002150 & 0.04823 & 22.43 \\
10 & 0.002150 & 0.04990 & 23.21 \\
15 & 0.002150 & 0.05012 & 23.31 \\
20 & 0.002150 & 0.05118 & 23.80 \\
25 & 0.002150 & 0.05199 & 24.18 \\
\hline
\end{tabular}

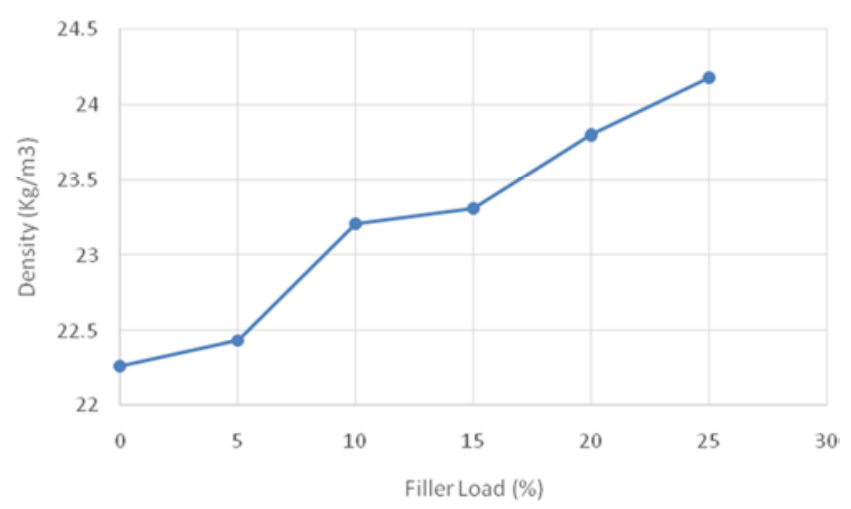

Figure 1. Effect of filler load on density of the foam samples.
Density is a key flexible polyurethane foam specification. The density of foam is defined as the ratio of mass per unit volume of the foam sample. In foam technology, density also measures the load bearing capacity and cost of any given foam sample. It is seen from the result in Table 2 and Figure 1 above, that the densities of the filled foams are higher than that of the pure polymer foam. This may be due to high content of fillers which would fill up more voids thus increasing the density. It could also be attributed to the enhanced mechanical properties exhibited by the fillers. More filler content will result to greater hardness of the foam. It will not easily collapse or sink after a heavy weight is placed on it [12]. Since, additives and fillers are used to increase foam density and simply to increase the ability of the foam to provide support. This is in agreement with some other works [13].

Table 3. Values for the Compression set.

\begin{tabular}{ll}
\hline Filler (\%) & \% Compression \\
\hline 0 & 3.8 \\
5 & 6.0 \\
10 & 8.0 \\
15 & 11.1 \\
20 & 11.5 \\
25 & 12.2 \\
\hline
\end{tabular}

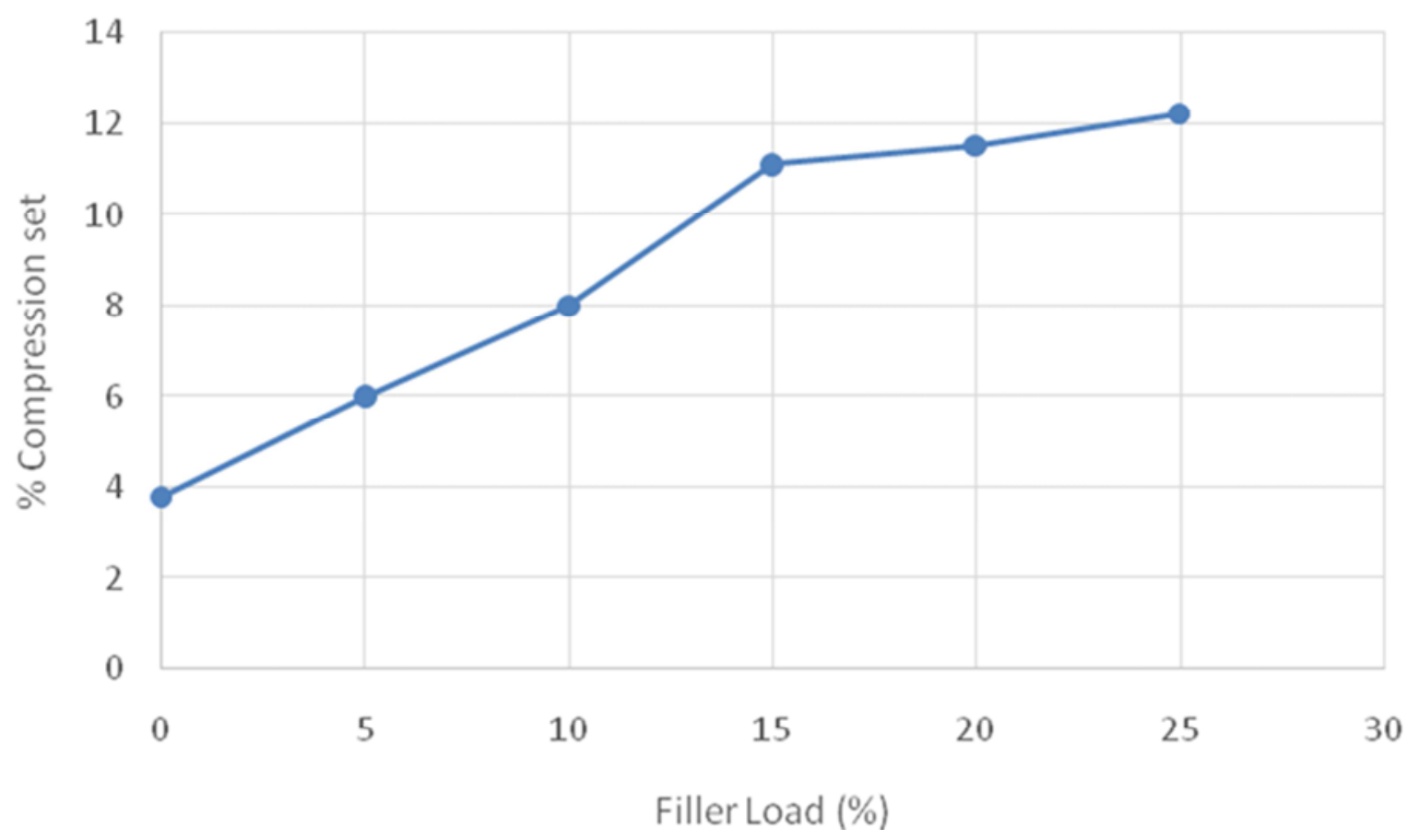

Figure 2. Effect of filler load on compression set of the foam samples.

It was seen from the result of the compression set test that as the filler load increased a gradual increase in the value of compression set was observed. This is as shown in Table 3 and Figure 2; this means that the foam samples will easily re-again its original height after a large weight is lifted from the foam. This is one of the qualities of good and durable foams. This could be attributed to the improved mechanical property displayed by the fillers. The fillers are all cellulosic materials and their reinforcing ability is therefore the load-carrying component in the produced foam samples [14]. 
Table 4. Values for Hardness test.

\begin{tabular}{lllll}
\hline Fillers Load (\%) & $\mathbf{2 5 \%}$ & $\mathbf{4 0 \%}$ & $\mathbf{6 5 \%}$ & Support factor 65\% IFD \\
\hline 0 & 134.6 & 153.1 & 212.3 & 1.6 \\
5 & 120.2 & 133.3 & 217.3 & 1.8 \\
10 & 91.4 & 98.7 & 154.7 & 1.7 \\
15 & 88.5 & 102.9 & 185.1 & 2.1 \\
20 & 96.3 & 114.1 & 192.9 & 2.0 \\
25 & 89.3 & 107.3 & 197.1 & 2.2 \\
\hline
\end{tabular}

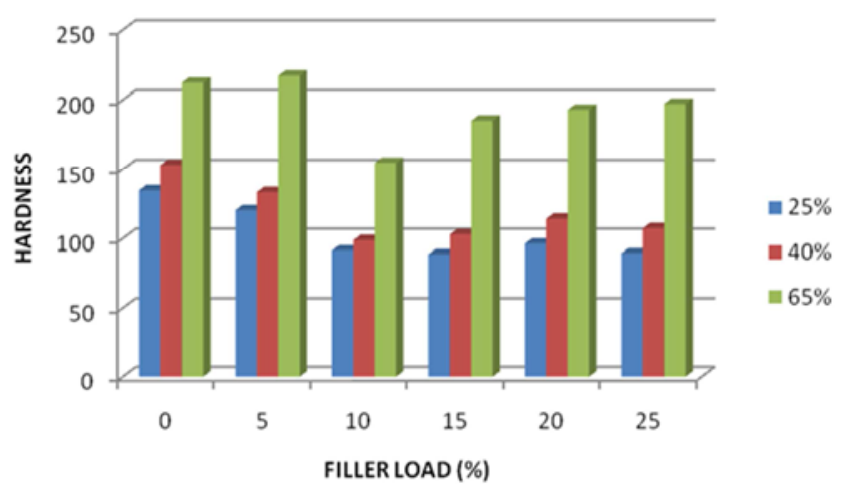

Figure 3. Effect of filler load on hardness of the foam samples.

The firmness of polyurethane foam is measured by a physical property called the indentation force deflection (IFD). The increase in the filler load was seen to have a positive impact on the samples by its increase on the hardness properties of the foam samples as shown in Table 4 and Figure 3. It means that the fillers will have a hard effect on the foam and would carry a lot of weight over a long period of time without collapsing immediately. These fillers are lingo-cellulosic materials and have been proved to be very strong and durable [14].

Table 5. Values for Tensile strength.

\begin{tabular}{ll}
\hline Filler Load $(\%)$ & Tensile strength $\left(\mathbf{k N} / \mathbf{M}^{\mathbf{2}}\right)$ \\
\hline 0 & 121.86 \\
5 & 112.74 \\
10 & 98.26 \\
15 & 89.44 \\
20 & 80.76 \\
25 & 71.52 \\
\hline
\end{tabular}

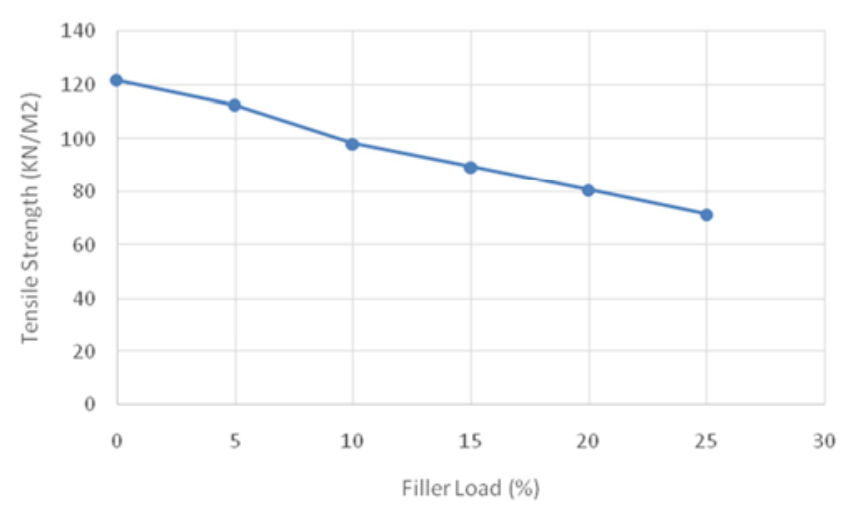

Figure 4. Effect of filler load on tensile strength of the foam samples.

It was observed that the tensile strength decreased as the filler load increased as seen in Table 5 and Figure 4. This may be due to poor interaction between the filler- polyurethane matrix. It could also be attributed to the voids created by the fillers in matrix phase. It is seen to be in accordance with the results of some other works done in the past $[10,14]$.

Table 6. Values for \% Elongation.

\begin{tabular}{ll}
\hline Filler Load (\%) & \% Elongation \\
\hline 0 & 164.20 \\
5 & 160.26 \\
10 & 153.75 \\
15 & 139.14 \\
20 & 121.68 \\
25 & 102.19 \\
\hline
\end{tabular}

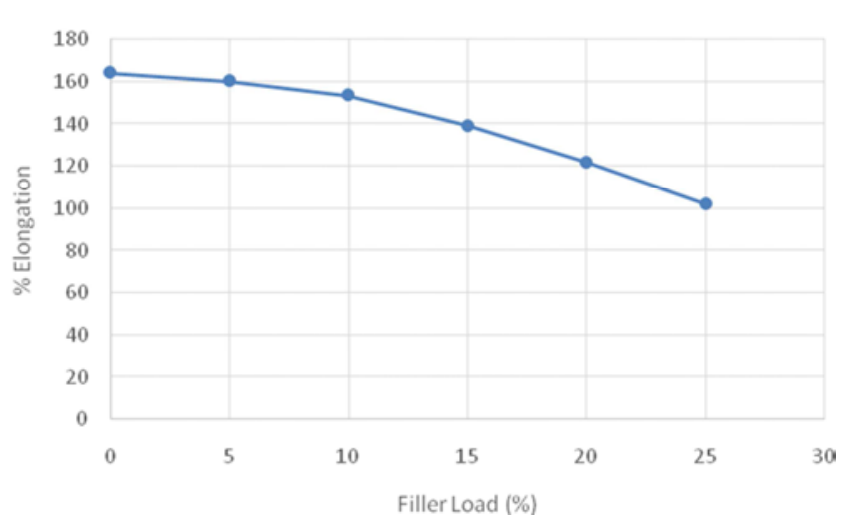

Figure 5. Effect of filler load on \%elongation at break of the foam samples.

From Table 6 and Figure 5; a decrease in the \%elongation at break was observed as the filler load increased. This could be as a result of its greater tendency to elongate with a lesser filler ratio than a higher ratio. The result is in agreement with the result of some researchers in the past [10].

Table 7. Values for Thermal conductivity.

\begin{tabular}{ll}
\hline Filler Load (\%) & Thermal conductivity $\left({ }^{\circ} \mathbf{C}\right)$ \\
\hline 0 & 150 \\
5 & 142 \\
10 & 131 \\
15 & 122 \\
20 & 109 \\
25 & 88 \\
\hline
\end{tabular}

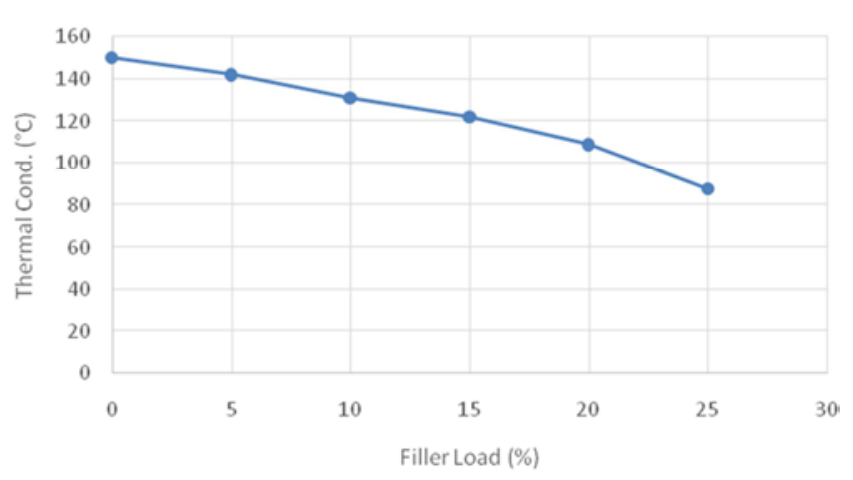

Figure 6. Effect of filler load on thermal conductivity of the foam samples.

The result of the thermal conductivity of the foam samples showed a decrease as the filler load increased as shown in Table 7 and Figure 6. It could be that the fillers having some 
fire retarding property. It means the foam is not easily flammable or will not support combustion. This is also in agreement with some researchers, as these fillers also have improved thermal and creep performance relative to the unfilled foam [15]. They can serve as fire retardant material.

Table 8. Foam Samples with Rising, Gellation and Curing Time.

\begin{tabular}{|c|c|c|c|c|}
\hline Filler (g) & Rising time (sec) & Gellation time (sec) & Curing time (hrs) & Creaming time (sec) \\
\hline 0 & 80 & $3-5$ & $18-24$ & $5-10$ \\
\hline 5 & 100 & $3-5$ & $18-24$ & $5-10$ \\
\hline 10 & 120 & $3-5$ & $18-24$ & $5-10$ \\
\hline 15 & 130 & $3-5$ & $18-24$ & $5-10$ \\
\hline 20 & 140 & $3-5$ & $18-24$ & $5-10$ \\
\hline 25 & 150 & $3-5$ & $18-24$ & $5-10$ \\
\hline
\end{tabular}

Creaming time is the first event measured, and occurs usually a short time after mixing, when the mixed liquids turn cloudy in appearance and the liquid begins to rise from its initial stable stage. It has been observed that the period lasts for about 12 seconds. As soon as generation of $\mathrm{CO}_{2}$ gas starts, the cream time is depend on the amount of amine used. High or high activity of amine starts the cream time.

Rising time is when the reacted foam has reached its largest volume, or maximum height. It is the period when the foam expansion has taken place such as: cell structure are foamed, gas reaction accompanied by generation of $\mathrm{CO}_{2}$ takes place and exploration of auxiliary blowing agent if present in formulation.

Curing time is when the foam is fully rise, the foam is initially soft gelatinous mass and the period between attainment of full rise and complete solidification of foam is the curing time. This is essentially the time most cross linking occurs.

It was observed from Table 8 above that the gellation and curing time of the foam samples produced were constant at different filler percentages while the rising time increased with increase in filler percentage.

\section{Conclusion}

The results of the physico-mechanical properties such as density and compression set showed an increasing trend with the increase in the filler. Elongation, tensile strength and thermal conductivity showed a decrease in their value as filler load increased while hardness (IFD) showed a slight random variation in the value as the filler also increased.

In general, the physico-mechanical analysis results of the synthesized foam samples showed that good quality foams for cushioning materials, mattresses, upholsteries, in fashion designing (as in shoulder pads) and special operations can be obtained from cellulosic materials in the range of $2 \%-5 \%$ application of filler (coconut husk and corn cob powder). Coconut husk and corn cob powder modified the characteristics and also added colour to a little extent to its appearance at a very low percentage. It is possible to substitute $\mathrm{CaCO}_{3}$ used in industries with coconut husk and corn cob, since it is much cheaper, readily available, and biodegradable and environmentally friendly than $\mathrm{CaCO}_{3}$.
They can decompose since the fillers are basically organic and agricultural wastes. More so, flammability of the foams can be reduced.

\section{References}

[1] Noah B. (2007) "Foam Polyurethane Technology: Paper presented at Lagos State University (Epe-campus) Nigeria, Pp 61-184.

[2] Billmeyer, Jnr. F. W. (2005); Textbook of Polymer Science; $3^{\text {rd }}$ edition, John Wiley andSons, Toronto, p.447.

[3] Saliba CC, Oréfice RL, Carneiro JRG, Duarte AK, Schneider WT, Fernandes MRF. Effect of the incorporation of a novel natural inorganic short fiber on the properties of polyurethane composites. Polym. Test. 2005; 24 (7): 819-824.

[4] Bartczak Z, Argon AS, Cohen RE, Weinberg M. Toughness mechanism in semi- crystalline polymer blends: II. Highdensity polyethylene toughened with calcium carbonate filler particles. Polymer 1999; 40 9): 2347-2365.

[5] Callister WD. Materials Science and Engineering: An Introduction. $5^{\text {th }}$ edition New York: John Wiley \& Sons; 2000.

[6] Nunes RCR, Fonseca JLC, Pereira MR. Polymer-filler interactions and mechanical properties of a polyurethane elastomer. Polym. Test. 2000; 19 (1): 93-103.

[7] Barma P, Rhodes MB, Salovey R. Mechanical properties of particulate-filled polyurethane foams. J. Appl. Phys. 1978; 49 (10): 4985-4991.

[8] Yeh, S. K. and Gupta, R. K. (2008); Improved Wood Plastic Composites through better processing. Composites: Part A. 39, 1694-1699.

[9] Bhatnager M. S. (2011); Text Book of Polymers; Chand Company Ltd., Ramnagar, New Delhi, Pp. 61-107, 96-201.

[10] Onuegbu T. U., Obianuko, N., Mbachu G. U. and Iloamaeke, I. M. (2010); The Effect of Animal Waste (Goat femur) as Filler in Flexible Polyether Foam. Journal of Basic Physical Research 1 (1): 5-8.

[11] Brain, J. (2009); "Polyurethane chemical and calculation" Inter-bur Publishers. London, Pp. 20-57.

[12] R. Gayathri, R., Vasanthakumari, R. and Padmanabhan, C. (2013); Sound absorption, Thermal and Mechanical behavior of Polyurethane foam modified with Nano silica, Nano clay and Crumb rubber fillers. International Journal of Scientific \& Engineering Research, 4 (5): 301-308. 
[13] Dalen, N. B., Ibrahim, A. Q., Adamu, H. M. and Nurudeen, A. A. (2014); Effects of $\mathrm{CaCO}_{3}$ and Kaolin Filler Loadings on Curing Rates of Polyurethane Foams, International Research Journal of Pure and Applied Chemistry. Diamond Print, Lagos.4 (6): Pp 691-709.

[14] Ruijun, G. Mohini, M. S and Samir, K. K. (2013); A Feasibility Study of Polyurethane Composite Foam with
Added Hardwood Pulp, Industrial Crops and Products. Checkmate Press, New York; 42 (2). Pp 273-279.

[15] Marcelo, A., Alvaro, C. and Jose, I. V. (2011); Esparto Wool as Reinforcement in Hybrid Polyurethane Composite Foams Industrial Crops and Products. Dove Publishers. Texas. 34 (3): 1641-1648. 